\title{
On the Relationship between Microhardness and Glass Transition Temperature of Some Amorphous Polymers
}

\author{
S. FAKIROV,* F. J. BALTÁ CALLEJA, M. KRUMOVA \\ Instituto de Estructura de la Materia, CSIC, Serrano 119, 28006 Madrid, Spain
}

Received 17 March 1998; revised 12 February 1999; accepted 16 February 1999

\begin{abstract}
On the basis of microhardness $(H)$ data measured at room temperature only for a number of polymers in the glassy state, a linear correlation between $H$ and the glass transition temperature $T_{g}$ has been found $\left(H=1.97 T_{g}-571\right)$. By means of this relationship, the deviation of the $H$ values from the additivity law for some multicomponent and/or multiphase polymeric systems can be accounted for. The latter usually contains a liquidlike soft component and/or phase with $T_{g}$ below room temperature. A completely different deformation mechanism in comparison to systems with $T_{g}$ above room temperature is invoked. A novel expression for the hardness of polymers in terms of crystallinity of the single components and/or phases, the $T_{g}$ values, and the mass fraction of each component is proposed. This expression permits the calculation of (i) the room-temperature $H$ value of amorphous polymers, mainly containing single bonds in the main chain, provided $T_{g}$ is known, and of (ii) the contribution of the soft liquidlike components (phases) to the hardness of the entire multiphase system. () 1999 John Wiley \& Sons, Inc. J Polym Sci B: Polym Phys 37: 1413-1419, 1999

Keywords: microhardness; glass transition temperature; additivity law; microhardness-depression effect
\end{abstract}

\section{INTRODUCTION}

Microindentation with a point indenter involving a deformation on a very small scale is one of the simplest ways to measure the mechanical properties of a material. ${ }^{1}$ The method uses a diamond pyramid that penetrates the surface of a specimen on application of a given load at a constant rate for a given time. Because of its simplicity, it has become a common technique to measure the micromechanical behavior of polymers and its correlation with microstructure., ${ }^{2,3}$ Microhardness is obtained by dividing the peak contact load by the projected area of impression, which is measured under the light microscope (imaging method).

* Permanent address: University of Sofia, Laboratory on Structure and Properties of Polymers, 1126 Sofia, Bulgaria

Correspondence to: F. J. Baltá Calleja (E-mail: embalta@ iem.csic.es)

Journal of Polymer Science: Part B: Polymer Physics, Vol. 37, 1413-1419 (1999) (ㄷ) 1999 John Wiley \& Sons, Inc.

CCC 0887-6266/99/131413-07
Hardness defined in this way is an indication of the irreversible plastic deformation process. The strain boundaries for plastic deformation below the indenter have been shown to depend on the morphology of the polymer (crystal size and perfection, degree of crystallinity, etc.). ${ }^{4}$ Typical loads of $10^{2} \mathrm{mN}$ when applied to the surface of a conventional polymer like poly(ethylene terephthalate) (PET) produce penetration depths of about 2-3 $\mu \mathrm{m}$. From a macroscopic point of view, $H$ is directly correlated to the yield stress of the material $\left(\sigma_{y}\right)$ through Tabor's relation $H \simeq k \sigma_{y}$, where $k \sim 2-3$. Tabor's relation is well substantiated for unoriented polymers when creep is minimized. ${ }^{4,5}$

The need to mechanically characterize very thin films and near surfaces has led to the development of ultramicro- and microindentation testers. ${ }^{6,7,8}$ Continuous load-displacement monitoring as the indenter is driven into and out from the surface substitutes the imaging method by 
the compliance one. Smaller loads can be applied $(0.1-1 \mathrm{mN})$, in this case leading to penetration depths within the submicron scale. In addition, the elastic recovery of the indentation on load removal can be studied. The hardness and elastic modulus of various polymers using the compliance method has been recently reported by various authors. ${ }^{8,9}$

The development of the scanning force microscope provides a new and improved technique for applying and measuring even smaller forces, and when applied to indentation measurements, opens up a new area of research. ${ }^{10}$ Experiments in the nanohardness range on polycarbonate (PC), polymethyl methacrylate (PMMA), and epoxy (EP) surfaces give hardness values higher than bulk hardness. ${ }^{11}$ This may be a genuine size effect or it may be the results of surface films. Clearly the preparation of the surface is extremely important when indentation may be as small as $20 \mathrm{~nm}$ in depth. Therefore, the conventional Vickers hardness test is more sensitive to bulk properties of the material and the SFM test primarily probes the mechanical properties of the surface.

Preceding studies show that microhardness determination using the imaging method is a promising technique for the microstructural investigation of multicomponent systems of known composition. $^{2}$ In these cases, $H$ can be very well described in terms of an additive system of independent hardness components.

The aims of the present study are twofold. (1) To discuss the existing correlation between $H$ and the glass transition temperature $\left(T_{g}\right)$ for amorphous polymers. (2) To explain the deviation of $H$ values from the hardness additivity law for multicomponent and multiphase systems containing components with a $T_{g}$ value below room temperature.

\section{Additivity Relationship}

It has been demonstrated ${ }^{12,13}$ that a number of glassy and semicrystalline polymers, copolymers, and blends obey the additivity law of independent hardness components $H_{i}$ :

$$
H=\sum_{i} H_{i} \varphi_{i}
$$

where $\varphi_{i}$ is the mass fraction of each component or phase. This relationship is of great value be- cause it offers the opportunity to characterize micromechanically components of a system which are not accessible to direct measurement, such as in the case of microfibrils in microfibrillar-reinforced composites. ${ }^{12}$

In other systems, as, for example, blends of high-density polyethylene with polypropylene, deviations from the additivity law were explained by the peculiarity in the morphological structure of crystallites formed, mostly related to changes in the surface-free energy. ${ }^{14}$

The same approach, when applied to poly(ether ester) (PEE) thermoplastic elastomers, ${ }^{15,16}$ failed to explain the large discrepancy found (up to 100 $\mathrm{MPa}$ when the measured $H$ values are in the range of 20 and $50 \mathrm{MPa}$ ) between the obtained and calculated values according to eq. (1). For this reason, we looked for other factors that could be responsible for this discrepancy.

Thermoplastic elastomers contain a hard segments phase $(h)$ and a very soft phase ( $s$ ) (with $T_{g}$ around $-50^{\circ} \mathrm{C}$ ), the latter being at room temperature in a liquid state and characterized by a viscosity closer to that of low molecular weight liquids rather than to solid amorphous polymers. For this reason, it is reasonably acceptable that such a liquidlike component would be characterized by a negligibly small microhardness value $H^{s}$ in the equation

$$
H=\varphi\left[H_{c}^{h} w_{c}+H_{a}^{h}\left(1-w_{c}\right)\right]+(1-\varphi) H^{s}
$$

where $\varphi$ is the mass fraction of hard segments, with $H_{c}^{h}$ and $H_{a}^{h}$ being the microhardness of the crystalline and amorphous phases, respectively, and $w_{c}$ being the degree of crystallinity of the hard segments. Assuming $H^{s}=0$, the calculations of $H$ according to eq. (2) for a series of PEE leads to differences between the measured and calculated $H$ value amounting to $40-64 \mathrm{MPa}$, depending on the soft segment composition, as will be shown later.

One may ask at this stage, what could be the reason for the failure of the additivity law? Obviously, one has to assume that for multicomponent and/or multiphase systems, provided that one of the components (phases) is characterized by a viscosity at room temperature typical for low molecular weight liquids, the microhardness behavior of the entire system should be different because the mechanism of the response to the applied external mechanical field is different from that when all the components have $T_{g}$ higher 


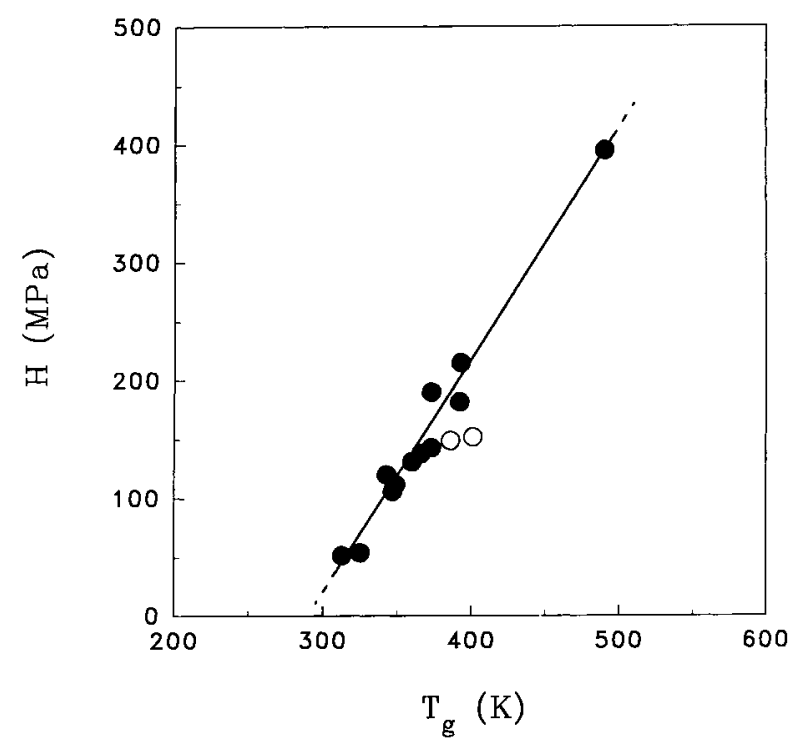

Figure 1. Relationship between the room temperature-measured microhardness $H$ and glass transition temperature $T_{g}$ for the amorphous polymers and copolymers listed in Table I. The open circles correspond to samples 9 and 13 in Table I (see text).

than room temperature. In the latter case, all the components deform plastically as a result of the applied external force. In the former case, in addition to the plastic deformation of the harder components, these also displace themselves within the soft (liquid) matrix in which they "float." The extent of this displacement depends on the viscosity of the matrix (the softer component and/or phase). For this reason, the harder components cannot display their inherent microhardness. The latter is reduced by the ability of the harder components to be displaced.

\section{Correlation between Microhardness and Glass Transition Temperature}

The question arises as to how one can quantitatively account for this microhardness-depression effect. As indicated before, the simple assumption that the soft segments contribute with a value $H$ $\simeq 0$ does not solve the problem. It is necessary to characterize the ability of the harder phase to displace itself within the soft matrix. This will depend on the viscosity of the matrix (i.e., the soft segments phase in the present case). Since $T_{g}$ and the viscosity are closely related, we looked for an analytical relationship between room temperature-measured microhardness of amorphous polymers and their $T_{g}$. Figure 1 illustrates this rela- tionship for some polymers (see Table I). Attention was paid to select mainly noncrystallizable polymers. For the crystallizable ones, for which there is no reliable evidence of the presence of a completely amorphous phase, as is the case for an example with polyamide 6 (PA6), the extrapolated to zero crystallinity value of $H$ was used. In this way, we attempt to avoid the very strong effect of even small amounts of the crystalline phase on $H$.

A fairly good linear relationship between the room-temperature $H$ and $T_{g}$ in the $T_{g}$ interval between room temperature and $250^{\circ} \mathrm{C}$ is found,

$$
H=k T_{g}+C
$$

where $C=-571 \mathrm{MPa}$ and $k=1.97 \mathrm{MPa} / \mathrm{K}$, the regression coefficient being 0.989 . These numerical values are obtained if one disregards the data for PC and PET/PC 10/90 [sample numbers 9 and 13 (open circles in Fig. 1), Table I, respectively] for reasons that will be discussed later. Nevertheless, if one considers the data for PC and PET/PC $10 / 90$, the numerical values of $C$ and $k$ still are very close to the cited ones.

The value of $C$ is physically meaningless. This value only reflects the fact that substances characterized by $T_{g}$ values below room temperature do not have the commonly used microhardness (i.e., the $H$ value measured at room temperature). To obtain physically meaningful $H$ values for these substances one should decrease the temperature at which the measurements have to be carried out in such a way that always $T_{\text {meas }}<T_{g}$. For this reason, $C$ has to be considered as a constant, which may help to describe in a better way the microhardness behavior of complex systems, as suggested in the present study.

\section{Application to Thermoplastic Elastomers}

Let us recall some characteristic features of the structure and morphology of thermoplastic elastomers of the PEE type that are closely related with the problem under discussion. The studied PEE samples represent polyblock copolymers consisting of hard poly(buthylene terephthalate) (PBT) segments and soft segment poly(glycols). The latter could be poly(tetramethylene glycol) (PTMG) or poly(ethylene glycol) (PEG). In a lab scale there also are synthesized poly(ester ether carbonate) (PEEC) comprising in the soft seg- 
Table I. Glass Transition Temperature $T_{g}$ and the Room Temperature-Measured Microhardness $H$ of the Polymers Used in the Plot of Figure 1

\begin{tabular}{|c|c|c|c|c|c|}
\hline Sample No. & Polymer & $\begin{array}{l}T_{g} \\
\left({ }^{\circ} \mathrm{C}\right)\end{array}$ & Ref. & $\begin{array}{c}\mathrm{H} \\
(\mathrm{MPa})\end{array}$ & Ref. \\
\hline 1 & Polyamide 6 (PA6) & 40 & 20 & $52 \pm 1^{\mathrm{a}}$ & 12 \\
\hline 2 & Poly(butylene terephthalate) (PBT) & 52 & 15 & $54 \pm 8^{\mathrm{a}}$ & 21 \\
\hline 3 & Poly(ethylene terephthalate) (PET) & 70 & 22 & $120 \pm 5^{\mathrm{a}}$ & 23 \\
\hline 4 & $\begin{array}{l}\text { Random copolymer of PET } \\
\text { and poly(1,4-cyclohexanediol terephthalate) } \\
(\mathrm{PCHT})(\mathrm{PET} / \mathrm{PCHT} 64 / 36)^{\mathbf{b}}\end{array}$ & 74 & 24 & $106 \pm 1$ & 24 \\
\hline 5 & Random copolymer of PET/PCHT 69/31 ${ }^{\mathrm{b}}$ & 76 & 24 & $111 \pm 1$ & 24 \\
\hline 6 & $\begin{array}{l}\text { Randomized copolymer of PET } \\
\text { and bisphenol-A-polycarbonate (PET/PC 70/30) }\end{array}$ & 87 & 13 & $131 \pm 3$ & 13 \\
\hline 7 & Randomized copolymer PET/PC 50/50 ${ }^{\mathrm{c}}$ & 93 & 13 & $138 \pm 3$ & 13 \\
\hline 8 & Randomized copolymer of PET/PC 30/70 & 100 & 13 & $143 \pm 3$ & 13 \\
\hline 9 & Randomized copolymer of PET/PC 10/90 ${ }^{\mathrm{c}}$ & 113 & 13 & $149 \pm 3$ & 13 \\
\hline 10 & Atactic polystyrene (a-PS) & 100 & 25 & $190 \pm 3$ & 25 \\
\hline 11 & Poly(ethylene naphthalene-2,6-dicarboxylate) (PEN) & 119 & 26 & $182 \pm 3$ & 26 \\
\hline 12 & Atactic poly(methyl methacrylate) (a-PMMA) & 120 & 22 & $215 \pm 5$ & 27 \\
\hline 13 & Bisphenol- $A$-polycarbonate (PC) & 128 & 13 & $152 \pm 3$ & 13 \\
\hline 14 & Gelatin (dry) & 217 & 28 & $395 \pm 8$ & 29 \\
\hline
\end{tabular}

a Value obtained by extrapolation to zero crystallinity.

${ }^{\mathrm{b}}$ Composition in mol \%.

${ }^{\mathrm{c}}$ Composition in wt \%. All the PET/PC copolymers are amorphous as shown by X-ray and DSC measurements and noncrystallizable, as follows from their composition. ${ }^{13}$

ments $\alpha, \omega$-dihydroxy-poly(alkylene carbonate) (PC). ${ }^{15}$

Starting from the basic knowledge about thermoplastic elastomers of the PEE type ${ }^{17,18}$ and from the structural characterization of the copolymers, comprising PTMG and $\mathrm{PC}^{15}$ or just $\mathrm{PEG}^{19}$ as soft segments, one may conclude that they are multiphase systems. Basically, they consist of four phases, crystalline PBT, amorphous PBT, crystalline PTMG (PEG), and amorphous PTMG (PEG). Since the poly(glycol) incorporated in the copolymer chains melts below room temperature, one deals practically with three phases. These phases are very well expressed on the DSC traces, the melting peak from the PBT crystals and two $T_{g}$ 's. The first $T_{g}$ step appears around $-50^{\circ} \mathrm{C}$ for the soft segments comprising only $\mathrm{PTMG}^{15}$ or PEG (for PEEC, the $T_{g}$ is up to $1^{\circ} \mathrm{C}$, depending on the PC amount ${ }^{15}$ ). The second $T_{g}$ value occurs around $50^{\circ} \mathrm{C}$, arising from the amorphous PBT regions.

Equation (3) offers the opportunity to characterize the contribution of the soft-segments phase in the case of PEE to the overall microhardness. For the quantitative evaluation of the microhardness-reduction effect, one has to replace $H^{s}$ in eq. (2) by eq. (3), using for $T_{g}$ the glass transition temperatures of the soft-segments phase $T_{g}^{s}$ (in $\mathrm{K})$ :

$$
\begin{aligned}
H=\varphi\left[H_{c}^{h} w_{c}+H_{a}^{h}\left(1-w_{c}\right)\right] & \\
& +(1-\varphi)\left(1.97 T_{g}^{s}-571\right)
\end{aligned}
$$

The calculation of the $H$ value for $\mathrm{PEE}$ and PEEC by means of eq. (4) gives data that are in good agreement with the measured values $H_{\text {exp }}$, as shown in Table II (samples 1-6).

Since the linear relationship seems to be valid in a rather wide temperature range (in the present case proven at least for $T_{g}$ values between -50 and $250^{\circ} \mathrm{C}$ ), eq. (4) can be rewritten in such a way, accounting also for the amorphous hardsegments phase:

$$
\begin{array}{r}
H=\varphi\left[H_{c}^{h} w_{c}+\left(1-w_{c}\right)\left(1.97 T_{g}^{h}-571\right)\right] \\
+(1-\varphi)\left(1.97 T_{g}^{s}-571\right)
\end{array}
$$

where $T_{g}^{h}$ is the $T_{g}$ of the amorphous hard-segments phase. The good agreement obtained between the $H_{\text {exp }}$ and $H_{\text {cal }}$ values is not surprising, in this case, as the contribution of the amorphous hard-segments phase has been already accounted 


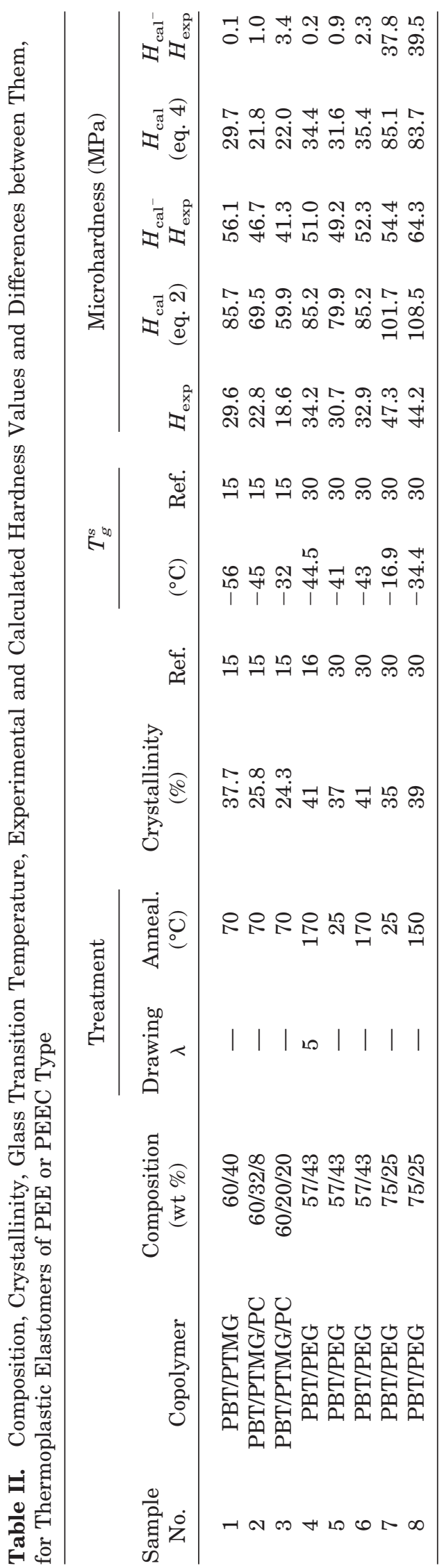

for in deriving the linear relationship (PBT belongs to the selected polymers for the plot in Fig. 1).

\section{DISCUSSION}

\section{Modified Additivity Law}

The advantage of incorporating $T_{g}$ in the additivity law, eq. (5), opens up the possibility of accounting for the contribution of any amorphous phase and/or component to the overall microhardness of a system, provided the $T_{g}$ value of this phase and/or component is known and its main chains contain mostly single bonds. Hence, for such systems, comprising more than one crystalline and/or amorphous phase with $T_{g}$ 's and mass fractions $T_{g i}$ and $\varphi_{i}$, respectively, the additivity law can be rewritten in the following way:

$$
\begin{array}{r}
H=\sum_{i} \varphi_{i} H_{c i} w_{c i}+\sum_{i} \varphi_{i}\left(1-w_{c i}\right)\left(1.97 T_{g i}-571\right), \\
\text { where } \sum_{i} \varphi_{i}=1
\end{array}
$$

In this form, the additivity law is applicable in contrast to the traditional one [eq. (1)] to multicomponent systems comprising liquidlike components at room temperature and displaying more complex deformation mechanisms in comparison to the case where all the amorphous components have $T_{g}$ above room temperature.

Let us come back to Table II, where data for other PEE with not such a good agreement between experimental and calculated $H$ values (Table II, samples 7 and 8) are presented. A possible explanation for the different behavior of the two groups of PEE samples could be the difference in their composition. Samples 1-6 (Table II) are characterized by hard/soft segment ratios of roughly 60/40, whereas the other ones (samples 7 and 8, Table II) present ratios of 75/25. The fact that in the second case the hard PBT segments are in a higher proportion than the soft ones may be the origin for another mechanism of response to the mechanical field, the PBT hard segments are presumably not floating anymore within the liquidlike matrix of soft segments.

\section{Influence of the Chemical Structure}

A peculiarity of the polymers listed in Table I is that their main chains comprise only single bonds 
(backbone chains, containing rings, as, for example, polyimides, disobey the linear relationship). This observation leads to the preliminary conclusion that the linear relationship is mostly valid for amorphous polymers distinguished by $T_{g}$, which is determined mainly by the rotation potential around single chemical bonds. Some of the data of Figure 1 are in favor of this assumption. For example, the points belonging to $\mathrm{PC}$ and the PET/PC (10/90) copolymer (containing the highest amount of PC in this series) (samples 9 and 13 , respectively) disobey to some extent the linear relationship between $H$ and $T_{g}$.

The above-mentioned deviation of data for PC and PET/PC (10/90) from the linear relationship (open circles in Fig. 1) can be explained by the fact that the chemical structure of these polymers (benzene rings dominate in the main-chain structure) significantly differs from that of polyolefines, polyesters, and polyamides, distinguished by single main-chain bonds. Obviously, for the same reason, data for polyarylates, containing even more benzene rings than PC, are far from the observed linear relationship and are not included in Table I and Figure 1, respectively.

The presence of benzene or other type of rings in the main chain drastically changes the rotation possibility around $\mathrm{C}-\mathrm{C}, \mathrm{C}-\mathrm{O}, \mathrm{C}-\mathrm{N}$, or other single bonds, and thus strongly affects the $T_{g}$ value.

Obviously, in the case when such aromatic or other rings are absent or single bonds strongly dominate, the relationship between $T_{g}$ and chain flexibility is more simple, and for this reason, the $T_{g}$ value can account for such basic properties as viscosity, the ability to pack more or less densely, and others.

The presence in the main chain of rings, particularly of aromatic type, results in the appearance of additional interaction forces between the neighboring chains and makes the relationship between $T_{g}$ and the chemical structure of the chains more complex. For this reason, one can expect, for polymers in which the $T_{g}$ is not determined mostly by the rotation potential around single chemical bonds, deviations from the linear relationship between $H$ and $T_{g}$. For the same reason, we prefer the values of $C$ and $k$ in eq. (3) to be taken without consideration of the data for PC and PET/PC 10/90 (Fig. 1, Table I), although strictly speaking, they do not seriously affect the values of $C$ and $k$.

Concerning the limitations of this relationship, it seems worth recalling that it is derived from data obtained from measurements carried out at room temperature. It is to be expected that the constant $C$ will depend on the temperature of measurement relative to $T_{g}$. This implies that to obtain a more fundamental relationship, one has to perform measurements at different temperatures and produce a $T_{g}$-scaled law of wider application.

\section{CONCLUSIONS}

Summarizing, one can conclude that owing to the linear relationship between the room temperature-measured $H$ and $T_{g}$ in a rather broad $T_{g}$ interval ( -50 up to $250^{\circ} \mathrm{C}$ ) covering a number of commonly used commercial polymers, mostly of polyolefine type but also including polyesters and polyamides, one can calculate the room-temperature microhardness value of many amorphous polymers, provided its $T_{g}$ is known and in the main chains dominate single bonds $\left(H=1.97 T_{g}\right.$ - 571). Furthermore, one can account for the contribution of the soft liquidlike components and/or phases (characterized by a negligibly small own microhardness at room temperature) to the microhardness of the entire system. It seems evident that the plastic deformation mechanism of such systems is different from that when all the components are in the solid state (i.e., with $T_{g}$ above room temperature).

Grateful acknowledgment is due to DGICYT (Grant PB94-0049), Spain, for the support of this investigation. The partial support of NATO (Grant No. CRG 920985 and NIG 951394) is also highly appreciated. It is a pleasure for one of us (S.F.) to acknowledge the tenure of a sabbatical grant from DGICYT, Spain.

\section{REFERENCES AND NOTES}

1. Tabor, D. The Hardness of Metals; Oxford C. Press: Glasgow, 1951.

2. Baltá Calleja, F. J. Trends Polym Sci 1994, 2, 419.

3. Deslandes, Y.; Alva Rosa, E.; Brisse, F.; Meneghini, T. J Mater Sci 1991, 22, 2769.

4. Baltá Calleja, F. J. Adv Polym Sci 1985, 66, 117.

5. Baltá Calleja, F. J.; Giri, L.; Ward, I. M.; Cansfield, D. L. M. J Mater Sci 1995, 30, 1139.

6. Doerner, M. F.; Nix, W. D. J Mater Res 1986, 1, 601.

7. Oliver, W. C.; Pharr, G. M. J Mater Res 1992, 7, 613. 
8. Briscoe, B. J.; Sebastian, K. S.; Sinha, S. K. Philos Mag A 1996, 74, 1159.

9. Ion, R. N.; Pollock, H. M.; Roques-Carmes, G. J Mater Sci 1990, 25, 1444.

10. Overny, R. M. Trends Polym Sci 1995, 3, 359.

11. Hamada, E.; Kaneko, R. Ultramicroscopy 1992, 42, 184.

12. Krumova, M.; Baltá Calleja, F. J.; Fakirov, S.; Evstatiev, M. J Mater Sci 1998, 33, 233.

13. Baltá Calleja, F. J.; Giri, L.; Ezquerra, T. A.; Fakirov, S.; Roslaniec, Z. J Macromol Sci, Phys 1997, $36,655$.

14. Baltá Calleja, F. J.; Santa Cruz, C.; Sawatari, C.; Asano, T. Macromolecules 1990, 23, 5352.

15. Baltá Calleja, F. J.; Fakirov, S.; Roslaniec, Z.; Krumova, M.; Ezquerra, T. A.; Rueda, D. R. J Macromol Sci, Phys 1998, 37, 219.

16. Apostolov, A. A.; Boneva, D.; Baltá Calleja, F. J.; Krumova, M.; Fakirov, S. J Macromol Sci, Phys 1998, 37, 543.

17. Schroeder, H.; Cela, R. in Encyclopedia of Polymer Science and Engineering, Vol. 12; Wiley: New York, 1988.

18. Legge, N. R.; Holder, G.; Schroeder, H. E.; Eds. Thermoplastic Elastomers, A Comprehensive Review; Hanser Publishers: Munich, 1987.

19. Stribeck, N.; Sapundjieva, D.; Denchev, Z.; Apostolov, A. A.; Zachmann, H. G.; Fakirov, S. Macromolecules 1997, 30, 1329.
20. Mark, J. E. Physical Properties of Polymers Handbook; American Institute of Physics: Woodbury, New York, 1996.

21. Giri, L.; Roslaniec, Z.; Ezquerra, T. A.; Baltá Calleja, F. J. J Macromol Sci, Phys 1997, B36, 335.

22. Brandrup, Y.; Immergut, E. H. Polymer Handbook, 3rd ed.; Wiley: New York, 1989.

23. Santa Cruz, C.; Baltá Calleja, F. J.; Zachmann, H. G.; Stribeck, N.; Asano, T. J Polym Sci, Polym Phys Ed 1991, 29, 819.

24. Krumova, M.; Karger-Kocsis, J.; Fakirov, S. Unpublished data.

25. Baltá Calleja, F. J.; García, M. C.; Rueda, D. R. Unpublished data.

26. Rueda, D. R.; Viksne, A.; Malers, L.; Baltá Calleja, F. J.; Zachmann, H. G. Macromol Chem Phys 1994, 195,3869 .

27. Ania, F.; Martínez-Salazar, J.; Baltá Calleja, F. J. J Mater Sci 1989, 24, 2934.

28. Rose, P. I. in Encyclopedia of Polymer Science and Engineering, Vol. 7; Wiley: New York, 1987; p 488.

29. Fakirov, S.; Cagiao, E.; Baltá Calleja, F. J.; Sapundjieva, D.; Vassileva, E. Int J Polym Mater to appear.

30. Fakirov, S.; Gogeva, T. Makromol Chem 1990, 191, 615. 